RESEARCH H IGHLIGHTS

B CELLS

\section{Phosphorylation initiates AID response}

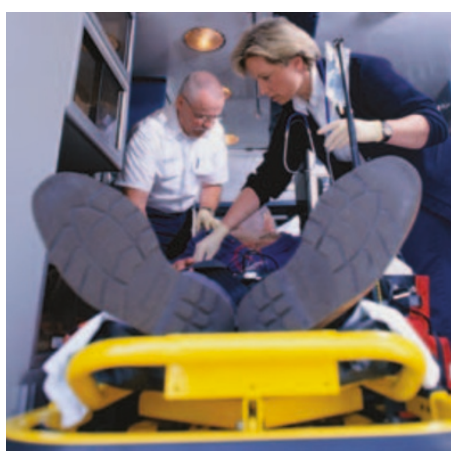

Activation-induced cytidine deaminase (AID) is required for antibody diversification through class-switch recombination (CSR) and somatic hypermutation (SHM). AID isolated from activated B cells has been shown to associate with replication protein A (RPA). This interaction requires phosphorylation of AID and is thought to direct AID to CSR and SHM target sequences in activated B cells. New research, published in Nature, now identifies cyclic-AMP-dependent protein kinase (PKA) as the enzyme that is responsible for phosphorylation of AID and regulation of AID-mediated CSR.

That AID isolated from activated $B$ cells can interact with RPA, whereas AID from 293 cells (which are nonlymphoid) engineered to express AID (denoted AID ${ }^{293}$ ) cannot, has been suggested to be important for the B-cell-specific targeting of AID to sites of CSR and SHM. Because the AID-RPA interaction requires phosphorylation of AID, Basu et al. set out to identify the protein kinase that is responsible for this in activated B cells. Nuclear extracts from activated B cells but not 293 cells could phosphorylate AID in vitro, indicating the presence of an AID protein kinase in nuclear extracts from activated B cells. Detailed analysis of these extracts showed that AIDprotein-kinase activity was associated with the catalytic subunit of PKA (denoted PKA ${ }_{\text {cat }}$ ) and that purified
PKA $_{\text {cat }}$ could phosphorylate AID ${ }^{293}$ in vitro. This phosphorylation conferred on $\mathrm{AID}^{293}$ the ability to deaminate double-stranded DNA (dsDNA) efficiently in vitro, a property that is associated with CSR and SHM (but freshly isolated AID $^{293}$ lacks this ability). Importantly, treatment with a PKA-specific inhibitor markedly impaired CSR in a B-cell clone that was activated to undergo CSR.

Mass-spectrometry analysis of AID from activated B cells showed that Ser38 and Tyr184 were phosphorylated, and Ser38 was found to be in a consensus site for phosphorylation by PKA. Thr27 was identified as an additional potential site for phosphorylation by PKA. AID mutants in which either Ser38 or Thr27 had been mutated to Ala were not phosphorylated by PKA, and they failed to interact with RPA and failed to mediate deamination of dsDNA. By contrast, an AID mutant in which Tyr184 had been substituted with Ala was phosphorylated and could mediate deamination of dsDNA. Consistent with these results, when each of these AID mutants was expressed by AID-deficient B cells induced to undergo CSR, only the AID mutant with the Tyr184Ala substitution could restore CSR.

These data indicate that PKA phosphorylates Ser38 of mouse AID, and this allows AID to interact with RPA and be targeted to sites of CSR. Because AID that is present in the cytoplasm of activated B cells is less efficient at deaminating dsDNA than is nuclear AID, the authors suggest that cytoplasmic AID is in an inactive state and that activation of PKA induces phosphorylation and thereby activation of AID such that it can enter the nucleus to mediate CSR.

Karen Honey

(2) References and links ORIGINAL RESEARCH PAPER Basu, U. et al. The AID antibody diversification enzyme is regulated by protein kinase A phosphorylation Nature 26 Oct 2005 (doi:10.1038/nature04255)

\section{IN BRIEF}

\section{IMMUNE REGULATION}

\section{Monocarboxylate transporter MCT1 is a target for} immunosuppression.

Murray, C. M. et al. Nature Chem. Biol. 30 Oct 2005 (doi:10.1038/nchembio744)

Previous studies identified a group of immunomodulatory compounds that were structurally distinct from other immunosuppressive agents. Murray et al. generated analogues of these compounds and showed that one inhibited T-cell proliferation in vivo. Surprisingly, whereas many immunosuppressive drugs (such as cyclosporin and rapamycin) inhibit T-cell cytokine production, this agent inhibited T-cell proliferation. Furthermore, its target was found to be monocarboxylate transporter 1 (MCT1), which is involved in the transport of monocarboxylates such as lactate and pyruvate across the plasma membrane. This agent could inhibit L-lactate efflux and thereby reduce the glycolytic rate of activated $\mathrm{T}$ cells, presumably (as the authors suggest) to rates that are insufficient to support rapid T-cell proliferation.

\section{IMMUNOLOGICAL SYNAPSES}

Cytotoxic granule polarization and cytolysis can occur without central supramolecular activation cluster formation in $\mathrm{CD}^{+}$effector $\mathrm{T}$ cells.

O’Keefe, J. P. \& Gajewski, T. F. J. Immunol. 175, 5581-5585 (2005)

Cell-surface receptors at the point of contact between an effector $\mathrm{CD}^{+} \mathrm{T}$ cell and a target cell segregate and cluster. Formation of a central supramolecular activation cluster (cSMAC) has been linked to granule exocytosis by, and cytotoxicity of, effector CD8 ${ }^{+}$ $\mathrm{T}$ cells. However, data in this report indicate that formation of a cSMAC is not required. First, the number of effector $\mathrm{CD}^{+} \mathrm{T}$ cells that formed a cSMAC after encountering a target cell was fewer than the number of cells in which granules polarized towards the point of cell-cell contact. Second, the number of effector $\mathrm{CD}^{+} \mathrm{T}$ cells that formed a cSMAC was increased by target-cell expression of CD80, but the number of cells in which granules polarized towards the site of cell-cell contact and that mediated target-cell lysis was similar in the presence or absence of CD80.

\section{CYTOKINES}

\section{Transcriptional repressor DREAM regulates} T-lymphocyte proliferation and cytokine gene expression.

Savignac, M. et al. EMBO J. 24, 3555-3564 (2005)

Savignac et al. show that downstream-regulatory-element antagonist modulator (DREAM), which is a transcriptional repressor that is released from DNA following $\mathrm{Ca}^{2+}$ signalling, is expressed by T cells and that its expression is downregulated after T-cell receptor (TCR) ligation. T cells isolated from transgenic mice expressing a dominant-active form of DREAM (which remains bound to DNA following $\mathrm{Ca}^{2+}$ signalling) showed decreased proliferation and cytokine production following TCR ligation. Conversely, when DREAM expression was decreased in wild-type splenocytes, an increase in the basal expression of mRNA encoding interleukin-2 (IL-2) and interferon- $\gamma$ (IFN- $\gamma$ ) was detected. Because DREAM was bound to the promoters of the genes encoding IL- 2 and IFN- $\gamma$, the authors suggest that DREAM has a role in regulating the basal expression of these cytokines. 